\title{
Intrinsic asymmetry parameter in nonmesonic hypernuclear decay: analytical proof of the independence on hypernucleus within an independent particle shell model
}

\author{
C. Barbero \\ Departamento de Física, Universidad Nacional de La Plata, C. C. 67, 1900 La Plata, Argentina
}

\author{
A. Mariano \\ Instituto de Física La Plata, CONICET, 1900 La Plata, Argentina
}

(Received on 05 December, 2008)

\begin{abstract}
We derive general analytical expressions for the evaluation of the intrinsic asymmetry parameter in nonmesonic hypernuclear weak decay, within an independent particle shell model framework. Present formalism is valid for even-even, even-odd and odd-odd core hypernucleus. A standard strangeness-changing weak transition potential with exchange of the complete meson octet $\left(\pi, \eta, K, \rho, \omega, K^{*}\right)$ is assumed. Very simple analytical formulas are obtained within the $s$-wave approximation when we restrict to the $\pi$ and $\pi+K$ exchange models, providing an useful and manageable tool to understand the origin of the discrepancies between theoretical and experimental results. Numerical values for ${ }_{\Lambda}^{4} \mathrm{He},{ }_{\Lambda}^{5} \mathrm{He},{ }_{\Lambda}^{11} \mathrm{~B},{ }_{\Lambda}^{12} \mathrm{C},{ }_{\Lambda}^{16} \mathrm{O},{ }_{\Lambda}^{17} \mathrm{O}$ and ${ }_{\Lambda}^{28} \mathrm{Si}$ hypernuclei are exhibited. Present formalism could be used to understand why new contributions to the exchange potential allow to obtain good agreement with data, as indicated in very recent works. The necessity of a consistent inclusion of: i) different modifications of the exchange potential (two-pion exchange, $a_{1}$ meson, $\Delta T=3 / 2$ terms coming from vector mesons, etc); ii) final state interactions on primary nucleons inside the residual nucleus and also correlations between the free emitted ones; and iii) configuration mixing in the final system, is remarked.
\end{abstract}

Keywords: Hypernuclear decay; Asymmetry parameter; One-meson-exchange; Independent particle shell model

\section{INTRODUCTION}

The free decay of a $\Lambda$ hyperon occurs almost exclusively through the mesonic mode, $\Lambda \rightarrow \pi N$, emerging the nucleon with a momentum of about $100 \mathrm{MeV} / \mathrm{c}$. Inside the nuclear medium $\left(p_{F} \sim 270 \mathrm{MeV} / \mathrm{c}\right.$ ) this mode is Pauli blocked and the weak decay is dominated by the nonmesonic channel, $\Lambda N \rightarrow N N$. The transitions receive contributions either from neutrons $(\Lambda n \rightarrow n n)$ and protons $(\Lambda p \rightarrow n p)$, with rates $\Gamma_{n}$ and $\Gamma_{p}$, respectively. For a long time, the experimental data for this process were restricted to the total nonmesonic decay rate, $\Gamma_{N M}$, and the neutron to proton ratio, $\Gamma_{n / p}=\Gamma_{n} / \Gamma_{p}$. The discrepancies between the theoretical results, $\Gamma_{n / p}^{\text {theor }} \simeq 0.1-0.5$, and the experimental data, $\Gamma_{n / p}^{e x p} \simeq 1$, have constituted an interesting puzzle for many years. In the light of recent developments [1, 2] and of new measurements [3]-[9] the experimental value of this ratio seems to be small and close to 0.5 , which could lead to think that the nonmesonic hypernuclear decay (NMHD) problem has been solved. However, discrepancies still exist between theory and experiment and also among various set of data. In fact, for example, experimental proton spectra measured in different laboratories are incompatible between them but also with those evaluated from theoretical models, as discussed carefully in the very recent work of the FINUDA Collaboration [10].

Additionally, in the last years, the results obtained at KEK [11]-[15] for another important observable, namely, the intrinsic asymmetry parameter, $a_{\Lambda}$, have been available. This is experimentally more demanding, as it requires measuring the asymmetry in the angular distribution of protons emitted in the decay of polarized hypernuclei. On the theoretical side, however, $a_{\Lambda}$ carries important new information, since it is determined by the interference terms between the parity-conserving (PC) and the parity-violating (PV) proton-induced transitions to final states with different isospins. One expects, therefore, that the asymmetry parameter will have more discriminating power to constrain the proposed mechanisms for NMHD. As known, the measurements of $a_{\Lambda}$ favor a negative value for ${ }_{\Lambda}^{12} C$ and a positive value for ${ }_{\Lambda}^{5} \mathrm{He}$ [11]-[15]. On the other hand, majority of the existing calculations based on strict one-meson exchange models (OMEM) [16]-[26] find values between -0.73 and -0.19 for ${ }_{\Lambda}^{5} \mathrm{He}[27,28]$ and, when results are available in the same model, very similar values for ${ }_{\Lambda}^{12} C$. Thus, there is now a renewed interest to solve this NMHD puzzle. A first application of effective field theory (EFT) to nonmesonic decay [29] and the inclusion of $\sigma$ meson exchange plus direct-quark interaction, which strongly violates the $\Delta T=1 / 2$ rule [30], have stressed that the scalar-isoscalar type of interactions are specially important in explaining the asymmetry. Quite recently, two relevant works have pointed to possible solutions via the inclusion of: i) a one-meson-exchange potential supplemented by a correlated plus uncorrelated chirally motivated two-pion

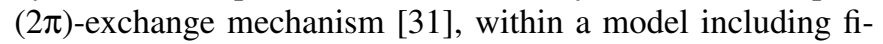
nal state interactions (FSI) for different cuts on the kinetic energy of the emitted nucleons; or ii) the $a_{1}$ meson exchange [32]. These works give some assessment in connection with the asymmetry problem. Nevertheless, due to the diversity of ways in which the puzzle seems that could be solved, it would be very useful to pursue in the analysis of the main degrees of freedom of finite hypernucleus nuclear structure models in order to establish a platform to study the mentioned new effects together and consistently.

A shell model (SM) formalism for the evaluation of the intrinsic asymmetry parameter has been developed quite recently in Ref. [23]. Additionally, the bridge between nuclear matter and finite hypernucleus formalisms has been established in Ref. [33], where it was shown that the finite hypernucleus formula reduces to the usual Nabetani-Ogaito-Sato-Kishimoto one [34] within the so-called $s$-wave approximation. Having in mind that the ${ }_{\Lambda}^{A} Z$ hypernucleus is considered as a $\Lambda$ particle coupled to an $A-1$ core, we need to distinguish between the cases with even-even, even-odd and odd-odd cores. The formalism introduced in [23] has been established only for even- 
even and even-odd core hypernucleus, and was applied specifically to ${ }_{\Lambda}^{5} \mathrm{He}$ and ${ }_{\Lambda}^{12} \mathrm{C}$.

Based in the actual status of NMHD problem it would be very useful to establish, within the SM framework, conclusions valid for all types of hypernuclei about theoretical predictions for asymmetry parameter. In particular, they could clearly give a guide to find the origin of discrepancies with experimental data. Motivated by this fact, we extend in the present work the formalism from [23] to the odd-odd core case, and obtain a very simple analytical expression for $a_{\Lambda}$ valid for all kind of hypernucleus. The aim of the present is not to solve the puzzle, but to explicitly remark the limitations of our formalism, and have certain control on it, to include consistently in the future the missing ingredients and decide which ones to add in order to obtain agreement with experiments.

This paper is organized as follows. In Sect. 2 the general formalism for $a_{\Lambda}$ is summarized. In Sect. 3 numerical results for ${ }_{\Lambda}^{4} \mathrm{He},{ }_{\Lambda}^{5} \mathrm{He},{ }_{\Lambda}^{11} \mathrm{~B},{ }_{\Lambda}^{12} \mathrm{C},{ }_{\Lambda}^{16} \mathrm{O},{ }_{\Lambda}^{17} \mathrm{O}$ and ${ }_{\Lambda}^{28} \mathrm{Si}$ hypernuclei are presented, and analytical formulas are obtained within the $s$ wave approximation, both for $\pi$ and $\pi+K$ exchange models. Sect. 4 contains conclusions and final remarks.

\section{FORMALISM}

The intrinsic asymmetry parameter for the proton induced NMHD, $\vec{\Lambda} p \rightarrow n p$, of an initial polarized hypernucleus (with spin $J_{I}$ and energy $\mathcal{E}_{I}$ ) to a residual nucleus (with spin $J_{F}$ and energy $\mathcal{E}_{F}$ ) plus two free nucleons (with momenta $\mathbf{p}_{1}$ and $\mathbf{p}_{2}$ ) will be calculated as [23]

$$
a_{\Lambda}=\left\{\begin{array}{cl}
A_{V}, & \text { for } J_{I}=J_{C}+1 / 2, \\
-\frac{J_{I}+1}{J_{I}} A_{V}, & \text { for } J_{I}=J_{C}-1 / 2,
\end{array}\right.
$$

where

$$
A_{V}=3 \sqrt{\frac{J_{I}}{J_{I}+1}} \frac{\sigma_{1}\left(J_{I}\right)}{\sigma_{0}\left(J_{I}\right)}
$$

with

$$
\begin{aligned}
\sigma_{\kappa}\left(J_{I}\right) & =\frac{1}{2}(4 \pi)^{4} \hat{J}_{I}^{3} \hat{\kappa}^{-1} \int d \cos \theta_{p_{1}} \int d F \sum_{S T T^{\prime}}(-)^{T+T^{\prime}} \\
& \times \sum_{l L \lambda J} \sum_{l^{\prime} L^{\prime} \lambda^{\prime} J^{\prime}} i^{-l^{\prime}-L^{\prime}-l-L}(-)^{\lambda+S+J+J^{\prime}+J_{I}+J_{F}} \hat{l} \hat{l}^{\prime} \hat{L} \hat{L^{\prime}} \hat{\lambda} \hat{\lambda}^{\prime} \hat{J} \hat{J}^{\prime} \\
& \times \sum_{k K}\left(l 0 l^{\prime} 0 \mid k 0\right)\left(L 0 L^{\prime} 0 \mid K 0\right)\left[Y_{k}\left(\theta_{p}, \pi\right) \otimes Y_{K}\left(\theta_{P}, 0\right)\right]_{\kappa 0} \\
& \times\left\{\begin{array}{lll}
J_{I} & \kappa & J_{I} \\
J & J_{F} & J^{\prime}
\end{array}\right\}\left\{\begin{array}{ccc}
\kappa & J^{\prime} & J \\
S & \lambda & \lambda^{\prime}
\end{array}\right\}\left\{\begin{array}{lll}
l & l^{\prime} & k \\
L & L^{\prime} & K \\
\lambda & \lambda^{\prime} & \kappa
\end{array}\right\} \\
& \times\left\langle p l P L \lambda S J T v_{F} J_{F} ; J_{I}|V| J_{I}\right\rangle \\
& \times\left\langle p l^{\prime} P L^{\prime} \lambda^{\prime} S J^{\prime} T^{\prime} v_{F} J_{F} ; J_{I}|V| J_{I}\right\rangle^{*} .
\end{aligned}
$$

We have defined

$$
\left\langle p l P L \lambda S J T v_{F} J_{F} ; J_{I}|V| J_{I}\right\rangle=\hat{J}_{I}^{-1} \sum_{\mathrm{j}_{N}} f_{J}\left(\mathrm{j}_{N}, \mathrm{v}_{F} J_{F}\right) \mathcal{M}\left(p l P L \lambda S J T ; \mathrm{j}_{\Lambda} \mathrm{j}_{N}\right)
$$

with $V$ being the transition potential, $\mathrm{j}_{\Lambda} \equiv n_{\Lambda} l_{\Lambda} j_{\Lambda} t_{\Lambda}$ and $\mathrm{j}_{N} \equiv n_{N} l_{N} j_{N} t_{N}$ the single-particle states for the lambda and nucleon, respectively (we assume that the $\Lambda$ particle behaves as a $\left|\frac{1}{2},-\frac{1}{2}\right\rangle$ isospin particle in the $1 s_{1 / 2}$ level) $p$ and $P$ the relative and center of mass momenta (see Eq. (15) from [23]) and $v_{F}$ specifying the remaining quantum numbers in the final state besides the nuclear spin. The factors are defined as

$$
f_{J}\left(\mathrm{j}_{N}, v_{F} J_{F}\right)=(-)^{2 J_{F}} \hat{J}_{I}\left\{\begin{array}{ccc}
J_{C} & J_{I} & j_{\Lambda} \\
J & j_{N} & J_{F}
\end{array}\right\}\left\langle J_{C} \| a_{\mathrm{j}_{N}}^{\dagger}|| v_{F} J_{F}\right\rangle,
$$

with $J_{C}$ being the core spin and the matrix element

$$
\mathcal{M}\left(p l P L \lambda S J T ; \mathrm{j}_{\Lambda} \mathrm{j}_{N}\right)=\frac{1}{\sqrt{2}}\left[1-(-)^{l+S+T}\right]\left(p l P L \lambda S J T|V| \mathrm{j}_{\Lambda} \mathrm{j}_{N} J\right) .
$$

Here, the compact notation has been introduced $(\hbar=c=1)$

$$
\int d F \ldots=2 \pi \sum_{v_{F} J_{F}} \int \frac{p_{2}^{2} d p_{2}}{(2 \pi)^{3}} \int \frac{p_{1}^{2} d p_{1}}{(2 \pi)^{3}} \delta\left(\frac{p_{1}^{2}}{2 M}+\frac{p_{2}^{2}}{2 M}+\frac{\left|\mathbf{p}_{1}+\mathbf{p}_{2}\right|^{2}}{2 M_{F}}-\Delta_{v_{F} J_{F}}\right) \ldots
$$

where $\Delta_{V_{F} J_{F}}=\mathcal{E}_{I}-\mathcal{E}_{F}-2 M_{N}$ is the released energy, being $M_{N}$ the nucleon mass. To get a formalism valid for all types of hypernucleus, we remark here that the difference between them is contained in the matrix element $\left\langle J_{C} \| a_{\mathrm{j}_{N}}^{\dagger}|| v_{F} J_{F}\right\rangle$, and thus in $f_{J}\left(\mathrm{j}_{N}, v_{F} J_{F}\right)$. Fig. 1 shows the initial and final states contributing to each case. As we work within the extreme in- 
dependent particle shell model (IPSM) the final state for the proton induced decay in the odd-odd case will be described as $\left|v_{F} J_{F}\right\rangle=\left|\left[\mathrm{j}_{n_{c}}^{-1}\left(\mathrm{j}_{p_{c}}^{-1} \mathrm{j}_{p}^{-1}\right) J_{1}\right] J_{F}\right\rangle$. Here $\left|J_{C}\right\rangle=\left|\left(\mathrm{j}_{n_{c}}^{-1} \mathrm{j}_{p_{c}}^{-1}\right) J_{C}\right\rangle$ represents the core, with $\mathrm{j}_{n_{c}} \equiv n_{n_{c}} l_{n_{c}} j_{n_{c}} t_{n_{c}}\left(\mathrm{j}_{p_{c}} \equiv n_{p_{c}} l_{p_{c}} j_{p_{c}} t_{p_{c}}\right)$ being the single-particle state of the neutron (proton) hole, and $j_{p}$ that of the outgoing proton. Thus, the extra label $v_{F}$ in the final states will be associated with the occupied single-proton states, $\mathrm{j}_{p}$, and the coupling quantum number $J_{1}$. Consequently, for a fixed single particle proton state, only one term contributes to the sum in Eq. (4), which suggests to rewrite our Eq. (7) as

$$
\int d F \cdots=\frac{1}{(2 \pi)^{5}} \sum_{\mathrm{j}_{p}} \int d F_{\mathrm{j}_{p}} \sum_{J_{1} J_{F}} \ldots
$$

where

$$
\begin{aligned}
& \int d F_{\mathrm{j}_{p}} \cdots=\int p_{2}^{2} d p_{2} \int p_{1}^{2} d p_{1} \\
& \times \delta\left(\frac{p_{1}^{2}}{2 M_{N}}+\frac{p_{2}^{2}}{2 M_{N}}+\frac{\left|\mathbf{p}_{1}+\mathbf{p}_{2}\right|^{2}}{2 M_{R}}-\Delta_{\mathrm{j}_{p}}\right) \ldots
\end{aligned}
$$

being $M_{R}$ the mass of the residual nucleus and $\Delta_{\mathrm{j}_{p}}=M_{\Lambda}-$ $M_{N}+\varepsilon_{\mathrm{j}_{p}}+\varepsilon_{j_{\Lambda}}$, with the single particle energies $\varepsilon_{\mathrm{j}_{p}}$ and $\varepsilon_{j_{\Lambda}}$ for proton and lambda particle (with mass $M_{\Lambda}$ ) respectively.

Thus, from Eqs. (3), (4) and (8) we can write

$$
\begin{aligned}
\sigma_{\kappa}\left(J_{I}\right) & =\frac{4}{\pi} \hat{J}_{I} \hat{\kappa}^{-1} \sum_{\mathrm{j}_{p}} \int d \cos \theta_{p_{1}} \int d F_{\mathrm{j}_{p}} \sum_{S T T^{\prime}}(-)^{T+T^{\prime}} \\
& \times \sum_{l L \lambda J^{\prime}} \sum_{l^{\prime} L^{\prime} \lambda^{\prime} J^{\prime}} i^{-l^{\prime}-L^{\prime}-l-L}(-)^{\lambda+S+J+J^{\prime}+J_{I}} \hat{l} \hat{l}^{\prime} \hat{L} \hat{L^{\prime}} \hat{\lambda} \hat{\lambda}^{\prime} \hat{J} \hat{J}^{\prime} \\
& \times \sum_{k K}\left(l 0 l^{\prime} 0 \mid k 0\right)\left(L 0 L^{\prime} 0 \mid K 0\right)\left[Y_{k}\left(\theta_{p}, \pi\right) \otimes Y_{K}\left(\theta_{P}, 0\right)\right]_{\kappa} 0 \\
& \times\left\{\begin{array}{ccc}
\kappa & J^{\prime} & J \\
S & \lambda & \lambda^{\prime}
\end{array}\right\}\left\{\begin{array}{ccc}
l & l^{\prime} & k \\
L & L^{\prime} & K \\
\lambda & \lambda^{\prime} & \kappa
\end{array}\right\} S_{2}\left(J, J^{\prime}, \mathrm{j}_{p}, \kappa\right) \\
& \times \mathcal{M}\left(p l P L \lambda S J T ; \mathrm{j}_{\Lambda} \mathrm{j}_{N}\right) \mathcal{M}^{*}\left(p l^{\prime} P L^{\prime} \lambda^{\prime} S J^{\prime} T^{\prime} ; \mathrm{j}_{\Lambda} \mathrm{j}_{N}\right),(10)
\end{aligned}
$$

where

$$
\mathcal{S}_{2}\left(J, J^{\prime}, \mathrm{j}_{p}, \kappa\right) \equiv \sum_{J_{1} J_{F}}(-)^{J_{F}}\left\{\begin{array}{ccc}
J_{I} & \kappa & J_{I} \\
J & J_{F} & J^{\prime}
\end{array}\right\} f_{J}\left(\mathrm{j}_{p}, J_{1} J_{F}\right) f_{J^{\prime}}\left(\mathrm{j}_{p}, J_{1} J_{F}\right)
$$

For a ${ }_{\Lambda}^{A} Z$ hypernucleus with odd-odd core, after some simple algebra we get ${ }^{1}$

$$
\begin{aligned}
f_{J}\left(\mathrm{j}_{p}, J_{1} J_{F}\right) & =(-)^{j_{n_{c}}+j_{p_{c}}+J_{C}+2 J_{F}}\left[1+(-)^{J_{1}} \delta_{\mathrm{j}_{p} \mathrm{j}_{p_{c}}}\right]^{1 / 2} \hat{J} \hat{J}_{I} \hat{J}_{C} \hat{J}_{1} \hat{J}_{F} \\
& \times\left\{\begin{array}{ccc}
J_{C} & J_{I} & j_{\Lambda} \\
J & j_{N} & J_{F}
\end{array}\right\}\left\{\begin{array}{lll}
j_{n_{c}} & j_{p_{c}} & J_{C} \\
j_{N} & J_{F} & J_{1}
\end{array}\right\} .
\end{aligned}
$$

Besides, noting that $\left|J_{C}\right\rangle=\left|\left(\mathrm{j}_{n_{c}}^{-1}\right) J_{C}\right\rangle$ and $\left|J_{F}\right\rangle=\left|\left(\mathrm{j}_{n_{c}}^{-1} \mathrm{j}_{p}^{-1}\right) J_{F}\right\rangle$ for even-odd core hypernucleus, we observe that the results in this case can be obtained from those of odd-odd core by choosing $\mathrm{j}_{p_{c}}=0$ (which means $j_{n_{c}}=J_{C}$ and $J_{1}=j_{p}$ ). Similarly, the results for even-even core hypernucleus are reproduced with

\footnotetext{
${ }^{1}$ It is important to remark here that the allowed $j$ values are limited by the fact that, in the $j_{1} j_{2}$-coupling we are using here, one needs to satisfy the condition $j+t=o d d$ for configurations with $j_{1}=j_{2}$ [35].
}

$\mathrm{j}_{p_{c}}=\mathrm{j}_{n_{c}}=0$ (which means $J_{C}=0$ and $J_{F}=\mathrm{j}_{p}$ ) in odd-odd core formulas. Thus, we have arrived to the general expression (12) for the $f_{J}\left(\mathrm{j}_{p}, J_{1} J_{F}\right)$ coefficients of an odd-odd hypernuclei, which includes the even-odd and even-even hypernuclei as particular cases.

Finally, using Eq. (35) from [23] we can rearrange the asymmetry parameter as

$$
a_{\Lambda}=\frac{\omega_{1}}{\omega_{0}} .
$$

where

$$
\begin{aligned}
\omega_{\kappa} & =8 \sqrt{2} \sum_{\mathrm{j}_{p}} \int d \cos \theta_{p_{1}} \int d F_{\mathrm{j}_{p}} \sum_{S T T^{\prime}}(-)^{T+T^{\prime}} \\
& \times \sum_{l L \lambda J} \sum_{l^{\prime} L^{\prime} \lambda^{\prime} J^{\prime}} i^{-l^{\prime}-L^{\prime}-l-L}(-)^{\lambda+S+\mathrm{j}_{p}+\frac{1}{2}} \hat{l} \hat{l}^{\prime} \hat{L} \hat{L^{\prime}} \hat{\lambda} \hat{\lambda}^{\prime} \hat{J}^{2} \hat{J}^{2} \\
& \times \sum_{k K}\left(l 0 l^{\prime} 0 \mid k 0\right)\left(L 0 L^{\prime} 0 \mid K 0\right)\left[Y_{k}\left(\theta_{p}, \pi\right) \otimes Y_{K}\left(\theta_{P}, 0\right)\right]_{\kappa 0} \\
& \times\left\{\begin{array}{ccc}
\kappa & J^{\prime} & J \\
S & \lambda & \lambda^{\prime}
\end{array}\right\}\left\{\begin{array}{ccc}
l & l^{\prime} & k \\
L & L^{\prime} & K \\
\lambda & \lambda^{\prime} & \kappa
\end{array}\right\} \mathcal{C}_{\mathrm{j}_{p} J J^{\prime} \kappa} \\
& \times \mathcal{M}\left(p l P L \lambda S J T ; \mathrm{j}_{\Lambda} \mathrm{j}_{p}\right) \mathcal{M}^{*}\left(p l^{\prime} P L^{\prime} \lambda^{\prime} S J^{\prime} T^{\prime} ; \mathrm{j}_{\Lambda} \mathrm{j}_{p}\right),
\end{aligned}
$$

with the coefficient

$$
\begin{aligned}
\mathcal{C}_{\mathrm{j}_{p} J J^{\prime} \kappa}= & \left\{\begin{array}{ccc}
\kappa & j_{\Lambda} & j_{\Lambda} \\
j_{p} & J & J^{\prime}
\end{array}\right\}+\delta_{\mathrm{j}_{p} \mathrm{j}_{p_{c}}} \hat{J}_{C}^{2}(-)^{j_{n_{C}}+j_{p}+J_{C}+2 J_{I}} \\
\times & \frac{\left\{\begin{array}{cccc}
j_{\Lambda} & J_{I} & J_{C} & j_{p} \\
J & \kappa & J^{\prime} & J_{C} \\
j_{p} & J_{I} & j_{n_{c}} & j_{\Lambda}
\end{array}\right\}}{\left\{\begin{array}{lll}
j_{\Lambda} & J_{I} & J_{C} \\
J_{I} & j_{\Lambda} & \kappa
\end{array}\right\}}
\end{aligned}
$$

\section{RESULTS}

Numerical computation for ${ }_{\Lambda}^{4} \mathrm{He},{ }_{\Lambda}^{5} \mathrm{He},{ }_{\Lambda}^{11} \mathrm{~B},{ }_{\Lambda}^{12} \mathrm{C},{ }_{\Lambda}^{16} \mathrm{O}$, ${ }_{\Lambda}^{17} \mathrm{O}$ and ${ }_{\Lambda}^{28} \mathrm{Si}$ hypernuclei has been performed. A standard strangeness-changing weak $\Lambda N \rightarrow N N$ transition potential comprising the exchange of the complete pseudoscalar and vector meson octets $\left(\pi, \eta, K, \rho, \omega, K^{*}\right)$ was adopted $[18,19]$. In the calculation, corrections due to kinematical effects related to the $\Lambda$-nucleon mass difference and the first-order nonlocality terms, carefully discussed in Ref. [20], have been taken into account. Also, in the present evaluation we include both: i) finite nucleon size effects which are phenomenologically implemented by a monopole form factor $\left(\Lambda_{M}^{2}-\mu_{M}^{2}\right) /\left(\Lambda_{M}^{2}+q^{2}\right)$, being $\Lambda_{M}$ the cutoff for the meson $M$ [36]; and ii) initial and final short range correlations which are simulated, respectively, by means of the correlation functions

$$
\begin{aligned}
& g_{\Lambda N}(r)=\left(1-e^{-r^{2} / \alpha^{2}}\right)^{2}+\beta r^{2} e^{-r^{2} / \gamma^{2}}, \\
& g_{N N}(r)=1-j_{0}\left(q_{c} r\right),
\end{aligned}
$$

with $\alpha=0.5 \mathrm{fm}, \beta=0.25 \mathrm{fm}^{-2}, \gamma=1.28 \mathrm{fm}$, and $q_{c}=3.93$ $\mathrm{fm}^{-1}$.

Table I shows the numerical results for the intrinsic asymmetry parameter. For the sake of comparison, available experimental data are exhibited in Table II. These results indicate that present model predicts a value for $a_{\Lambda}$ approximately 
independent of the considered hypernucleus, which is large and negative. This is in contradiction with data, which give a small and positive central value for ${ }_{\Lambda}^{5} \mathrm{He}$. As discussed in Refs. $[19,21,28]$ the inclusion of FSI effects (due to both, the propagation of primary emitted nucleons inside the residual nucleus, simulated through a Monte Carlo code, and the interaction between the free emitted nucleons, treated through the solution of the scattering equation) could give certain dependence with the hypernucleus. In spite that they are not included within the present plain IPSM, it will be very useful to understand in detail the origin of this similarity between our theoretical predictions for all considered hypernuclei. This will provide a tool to discriminate in the future between different candidates (FSI, $2 \pi$ exchange, $a_{1}$ meson, etc) to be relevant ingredients in getting agreement with data. Thus, we follow the procedure adopted in Refs. $[33,36]$ to generate an approximated expression for $\omega_{\kappa}$ retaining only the dominant contribution coming from tensor $\left(\mathrm{T}^{20}\right)$ scalar $\left(\mathrm{S}^{0}\right)$ and $\mathrm{PV}\left(\mathrm{P}^{10}\right)$ matrix elements within $\pi$ and $\pi+K$ exchange models. After a very simple algebra we can rewrite Eq. (14) as

$$
\omega_{\kappa}^{\pi+K}=\frac{8}{\sqrt{\pi}} \int d \cos \theta_{p_{1}} \int d F_{\mathrm{j}_{p}} I_{\kappa}^{\pi+K}(p, P) Y_{\kappa} 0\left(\theta_{p}, 0\right),
$$

where

$$
\begin{aligned}
I_{0}^{\pi+K} & =\left(c_{1}+r_{1} R+w_{1} W\right)\left(\mathrm{S}_{K_{0}}^{0}\right)^{2}+\left(c_{2}+r_{2} R+w_{2} W\right)\left(\mathrm{S}_{1}^{0}\right)^{2} \\
& +\left(c_{3}+r_{3} R+w_{3} W\right) \mathrm{S}_{K_{0}}^{0} \mathrm{~S}_{1}^{0}+\left(c_{4}+r_{4} R+w_{4} W\right)\left(\mathrm{T}_{K_{0}}^{20}\right)^{2} \\
& +\left(c_{5}+r_{5} R+w_{5} W\right)\left(\mathrm{T}_{1}^{20}\right)^{2}+\left(c_{6}+r_{6} R+w_{6} W\right) \mathrm{T}_{K_{0}}^{20} \mathrm{~T}_{1}^{20} \\
& +\left(c_{7}+r_{7} R+w_{7} W\right)\left(\mathrm{P}_{K_{0}}^{10}\right)^{2}+\left(c_{8}+r_{8} R+w_{8} W\right)\left(\mathrm{P}_{\pi}^{10}\right)^{2} \\
& +\left(c_{9}+r_{9} R+w_{9} W\right)\left(\mathrm{P}_{K_{1}}^{10}\right)^{2} \\
& +\left(c_{10}+r_{10} R+w_{10} W\right) \mathrm{P}_{K_{0}}^{10} \mathrm{P}_{\pi}^{10} \\
& +\left(c_{11}+r_{11} R+w_{11} W\right) \mathrm{P}_{K_{0}}^{10} \mathrm{P}_{K_{1}}^{10} \\
& +\left(c_{12} r_{12} R+w_{12} W\right) \mathrm{P}_{\pi}^{10} \mathrm{P}_{K_{1}}^{10},
\end{aligned}
$$

and

$$
\begin{aligned}
I_{1}^{\pi+K} & =\left(c_{1}+\mathrm{r}_{1} R+\mathrm{w}_{1} W\right) \mathrm{S}_{K_{0}}^{0} \mathrm{P}_{K_{0}}^{10}+\left(\mathrm{c}_{2}+\mathrm{r}_{2} R+\mathrm{w}_{2} W\right) \mathrm{S}_{K_{0}}^{0} \mathrm{P}_{K_{1}}^{10} \\
& +\left(c_{3}+\mathrm{r}_{3} R+\mathrm{w}_{3} W\right) \mathrm{S}_{K_{0}}^{0} \mathrm{P}_{\pi}^{10}+\left(\mathrm{c}_{4}+\mathrm{r}_{4} R+\mathrm{w}_{4} W\right) \mathrm{S}_{1}^{0} \mathrm{P}_{K_{0}}^{10} \\
& +\left(\mathrm{c}_{5}+\mathrm{r}_{5} R+\mathrm{w}_{5} W\right) \mathrm{S}_{1}^{0} \mathrm{P}_{K_{1}}^{10}+\left(\mathrm{c}_{6}+\mathrm{r}_{6} R+\mathrm{w}_{6} W\right) \mathrm{S}_{1}^{0} \mathrm{P}_{\pi}^{10} \\
& +\left(\mathrm{c}_{7}+\mathrm{r}_{7} R+\mathrm{w}_{7} W\right) \mathrm{T}_{K_{0}}^{20} \mathrm{P}_{K_{0}}^{10}+\left(\mathrm{c}_{8}+\mathrm{r}_{8} R+\mathrm{w}_{8} W\right) \mathrm{T}_{K_{0}}^{20} \mathrm{P}_{K_{1}}^{10} \\
& +\left(c_{9}+\mathrm{r}_{9} R+\mathrm{w}_{9} W\right) \mathrm{T}_{K_{0}}^{20} \mathrm{P}_{\pi}^{10} \\
& +\left(c_{10}+\mathrm{r}_{10} R+\mathrm{w}_{10} W\right) \mathrm{T}_{1}^{20} \mathrm{P}_{K_{0}}^{10} \\
& +\left(c_{11}+\mathrm{r}_{11} R+\mathrm{w}_{11} W\right) \mathrm{T}_{1}^{20} \mathrm{P}_{K_{1}}^{10} \\
& +\left(c_{12}+\mathrm{r}_{12} R+\mathrm{w}_{12} W\right) \mathrm{T}_{1}^{20} \mathrm{P}_{\pi}^{10}
\end{aligned}
$$

Here the nuclear moments $S^{0}, T^{20}$ and $P^{10}$ depend on both $p$ and $P$ momenta (see Eqs. (4.11), (4.13), (4.14), (4.16) and appendix from [36] for the detailed definition) and we have introduced the ratios between the center of mass matrix elements:

$R=\left[\frac{(P 1 \mid 11)}{(P 0 \mid 10)}\right]^{2}=\frac{(b P)^{2}}{3}, \quad W=\left[\frac{(P 2 \mid 12)}{(P 0 \mid 10)}\right]^{2}=\frac{(b P)^{4}}{15}$,

with $b$ being the harmonic oscillator parameter (we employ the oscillator length corresponding to the energy $\hbar \omega=45 A^{-1 / 3}$ $\left.25 A^{-2 / 3} \mathrm{MeV}[36]\right)$.

The values of the $L=0$ coefficients $c_{i}$ and $c_{i}$ are given in Table III, being the same for all hypernuclei.

For hypernuclei with cores with full proton shells (see Fig. 1) we obtain

$$
\begin{aligned}
& r_{i}=\left\{\begin{aligned}
c_{i} & \text { for }{ }_{\Lambda}^{12} C \\
\frac{3}{2} c_{i} & \text { for }{ }_{\Lambda}^{16} O,{ }_{\Lambda}^{17} O \text { and }{ }_{\Lambda}^{28} \mathrm{Si} ; \\
0 & \text { otherwise }
\end{aligned}\right. \\
& \mathrm{r}_{i}=\left\{\begin{aligned}
\mathrm{c}_{i} & \text { for }{ }_{\Lambda}^{12} \mathrm{C} \\
\frac{3}{2} \mathrm{c}_{i} & \text { for }{ }_{\Lambda}^{16} O,{ }_{\Lambda}^{17} O \text { and }{ }_{\Lambda}^{28} \mathrm{Si}, \\
0 & \text { otherwise }
\end{aligned}\right.
\end{aligned}
$$

for $L=1$ matrix elements, and

$w_{i}=\left\{\begin{array}{rl}\frac{3}{4} c_{i} & \text { for }{ }_{\Lambda}^{28} S i \\ 0 & \text { otherwise }\end{array} ; \quad w_{i}=\left\{\begin{array}{rl}\frac{3}{4} c_{i} & \text { for }{ }_{\Lambda}^{28} S i \\ 0 & \text { otherwise }\end{array}\right.\right.$,

for $L=2$ ones. These results show that $r_{i} / c_{i}=\mathrm{r}_{j} / \mathrm{c}_{j}$ and $w_{i} / c_{i}=\mathrm{w}_{j} / \mathrm{c}_{j}$ (independently of the $i, j$ values) for each eveneven or even-odd hypernuclei. One interesting question which has not been answered until this moment is: why is it so? To find the answer, we have evaluated separately the contribution of each single particle state to each one of the coefficients. We have found that the contribution of each single particle states is always a factor times the contribution of the $1 s_{1 / 2}$ level. In fact, this factor holds $\frac{1}{2}$ for $1 p_{1 / 2}, 1$ for $1 p_{3 / 2}$, and $\frac{3}{4}$ for $1 d_{5 / 2}$ states, as can be also checked from our numerical results shown in Table I. Thus, because: i) we are working within the plain IPSM, in which the final states consist only of unperturbed $\mathrm{j}_{p}^{-1}$ hole states (without the inclusion of more complicated configurations taken into account excitations of the core); ii) we do not include FSI effects mentioned previously; and iii) we assume an exchange potential including only the contribution of $\pi, \eta, K, \rho, \omega$ and $K^{*}$ mesons, there is no interference between different single particle states, which leads directly to the results exhibit in Eqs. (21) and (22). As a consequence, the contribution of the $1 s_{1 / 2}$ state factorizes, with the same factor in $\omega_{0}$ and $\omega_{1}$. Next, if we adopt the $s$-wave approximation discussed in Ref. [33], which mainly consists in considering that: i) the liberated energy in (9) is approximated by $\Delta_{\mathrm{j}_{p}} \simeq \Delta \equiv M_{\Lambda}-M_{N}$; ii) the decay is basically back to back $\left(Y_{\kappa 0}(0,0)=\frac{\hat{\kappa}}{\sqrt{4 \pi}}\right)$; and iii) the matrix elements $\mathrm{T}^{20}$, $\mathrm{S}^{0}$ and $\mathrm{P}^{10}$ are computed at $p \simeq p_{\Delta} \equiv \sqrt{M_{N} \Delta}$ and then when $I_{\kappa}^{\pi+K}$ (Eqs.(18) and (19)) is replaced in Eq.(17) they can be factorized out the integral, we arrive to

$$
a_{\Lambda}^{\pi+K} \simeq-\frac{2\left[2\left(3 \mathrm{~T}_{1}^{20}-\mathrm{T}_{K_{0}}^{20}\right) \mathrm{P}_{\pi}^{10}-3\left(\mathrm{P}_{\pi}^{10}-\mathrm{P}_{K_{0}}^{10}\right) \mathrm{S}_{1}^{0}-\left(3 \mathrm{P}_{K_{1}}^{10}+2 \mathrm{P}_{\pi}^{10}\right) \mathrm{S}_{K_{0}}^{0}\right]}{6\left(3 \mathrm{~T}_{1}^{20}-\mathrm{T}_{K_{0}}^{20}\right)^{2}+3\left(\mathrm{P}_{\pi}^{10}\right)^{2}+3\left(\mathrm{P}_{K_{1}}^{10}\right)^{2}+2 \mathrm{P}_{\pi}^{10}\left(2 \mathrm{P}_{K_{1}}^{10}-\mathrm{P}_{K_{0}}^{10}\right)+\left(\mathrm{P}_{K_{0}}^{10}\right)^{2}+9\left(\mathrm{~S}_{1}^{0}\right)^{2}+3\left(\mathrm{~S}_{K_{0}}^{0}\right)^{2}}
$$


for ${ }_{\Lambda}^{4} \mathrm{He},{ }_{\Lambda}^{5} \mathrm{He},{ }_{\Lambda}^{12} \mathrm{C},{ }_{\Lambda}^{16} \mathrm{O},{ }_{\Lambda}^{17} \mathrm{O}$ and ${ }_{\Lambda}^{28} \mathrm{Si}$, which are all hypernuclei with full proton shells.

On the other side, when we have an incomplete core proton shell, as happens with ${ }_{\Lambda}^{11} \mathrm{~B}$, we get the coefficients presented in Table IV. Following the same procedure as before for the eveneven and even-odd core cases, we observe that there is not an exact factorization, due to the vacancies in the proton shell, i.e., the value of the ratios $r_{i} / c_{i}$ and $\mathrm{r}_{j} / \mathrm{c}_{j}$ depend now on the $i, j$ indexes. To simplify still more the analysis, we will adopt now the one-pion-exchange model (OPEM) [41-43] which describes qualitatively the physics involved in NMHD, in spite that the contribution of mesons heavier than pion are important in getting an accurate description of this decay. Within this model, the $s$-wave approximation leads to

$$
a_{\Lambda}^{\pi} \simeq-\frac{4\left(1+\frac{3}{4} R\right) T_{\pi}^{20} P_{\pi}^{10}}{18\left(1+\frac{23}{36} R\right)\left(T_{\pi}^{20}\right)^{2}+\left(1+\frac{73}{108} R\right)\left(P_{\pi}^{10}\right)^{2}} .
$$

This result shows explicitly that the R-dependent factor is not exactly the same for all the terms, as was mentioned before, for ${ }_{\Lambda}^{11} \mathrm{~B}$. This indicates that nuclear structure modifies the factorization, in spite that the final effect on the observables could be moderate mainly because of the rather high exchanged momentum $(\sim 400 \mathrm{MeV} / \mathrm{c})$. With a good approximation we can consider that $\frac{3}{4} \simeq \frac{23}{36} \simeq \frac{73}{108}$ (in agreement with this, from the Table I we see that the $1 p_{3 / 2}$ shell contribution is approximately 0.7 times the $1 s_{1 / 2}$ one) which leads to

$$
a_{\Lambda}^{\pi} \simeq-\frac{4 T_{\pi}^{20} P_{\pi}^{10}}{18\left(T_{\pi}^{20}\right)^{2}+\left(P_{\pi}^{10}\right)^{2}} .
$$

This result is of general validity, in the sense that it holds for even-even, even-odd and odd-odd hypernuclei (see also our Eq. (23)). Thus, we have extended the results obtained in Ref. [33]: i) within the present IPSM formalism, which comprises the exchange of the complete meson octet, the instrinsic asymmetry parameter will be roughly independent of the considered hypernucleus; ii) the large and negative value for $a_{\Lambda}$ is mainly due to the interplay between the PC tensor $\left(\mathrm{T}_{\pi}^{20}\right)$ and PV dipole $\left(\mathrm{P}_{\pi}^{10}\right)$ matrix elements. Our results indicate that an improvement of the IPSM is required. The present paper establishes and analyzes the limitations of a shell model formalism for the evaluation of $a_{\Lambda}$, valid for all kind of hypernuclei, on which could be included missing ingredients in the future. Particularly, it will be interesting to analyze the effects of: i) mixing between the final states considered in the IPSM and/or with more complex configurations through the residual nuclear interaction; ii) modifications of the exchange potential, as for example including $2 \pi$-exchange [31], axial vector $a_{1}$-meson [32], $\Delta T=3 / 2$ terms coming from vector mesons, etc, and the interplay between them; iii) final state interactions. Referred to the first point, we expect only a moderate contribution because of high momentum transferred. However, we believe that it would be interesting to study additional nuclear structure effects in detail in the future. On the other side, in view of the recent relevant works from [31, 32], it would be very interesting and indispensable to decide which one of the possible modifications to the exchange potential is more relevant, and what happens if one include both $\left(2 \pi\right.$ and $\left.a_{1}\right)$ simultaneously. As mentioned, this kind of studies could be performed with the present formalism as the starting in the future. Additionally, in view of the large error bars exhibited by the data, it will be very useful to have precise experimental values for different hypernuclei (more than ${ }_{\Lambda}^{5} \mathrm{He},{ }_{\Lambda}^{11} \mathrm{~B}$ and ${ }_{\Lambda}^{12} \mathrm{C}$ ). Thus, it will be possible differentiate the effects resulting from the different modifications to the IPSM.

\section{SUMMARIZING CONCLUSIONS}

We have derived general analytical expressions for the evaluation of the intrinsic asymmetry parameter $a_{\Lambda}$ in NMHD, within an IPSM framework, adopting the OMEM potential. Our formulae is valid for even-even, even-odd and odd-odd core hypernuclei. In agreement with other evaluations based in the OMEM, we have shown that the model predicts a large and negative value for this observable, independently of the considered hypernucleus. In order to understand the origin of this similarity in the theoretical results, we have obtained by the first time simple analytical expressions within the $s$-wave approximation, both for $\pi$ and $\pi+K$ exchange models, valid for all hypernucleus.

We conclude that to get compatibility between theoretical results and experimental data it will be necessary to improve the model adding other not included ingredients. Between them, we propose as an interesting issue the analysis, within a finite nucleus framework, of nuclear residual interactions effects in the initial and final systems. This will allow interference terms between the contribution of different single particle states and probably will eliminate the factorization effect mentioned above, leading to a dependence of $a_{\Lambda}$ with the hypernucleus. Because we hope that this break of the factorization originated from nuclear structure effects will not have a relevant weight on the final value of $a_{\Lambda}$ due to the high exchanged momentum, another possibilities should be also analyzed. Between them and in agreement with recent contributions from Refs. [31, 32], a plausible possibility to avoid the mentioned simplification between the factorization in $\omega_{0}$ and $\omega_{1}$ could be the modification of the exchange potential. This could be done, for example, adding $2 \pi$-exchange mechanism, and/or $a_{1}$ meson contribution and/or $\Delta T=3 / 2$ due to vector mesons to the exchange potential. Particularly, the $2 \pi$ effect on the asymmetry parameter has been recently analyzed in Ref. [31] where the authors show that an agreement between theoretical and experimental results is achieved. Also, in Ref. [32] has been shown that the contribution of $a_{1}$ meson leads to numerical results being within the error bars. It is important to remark one more time that the calculation from Ref. [31] includes FSI effects as well, an effect that eventually could also to affect the previously mentioned factorization. Thus, present paper provides a formalism to analyze in the future what specific aspects of those exchanges give rise to substantial deviations from the value $\sim-0.5$. 


\section{ACKNOWLEDGMENTS}
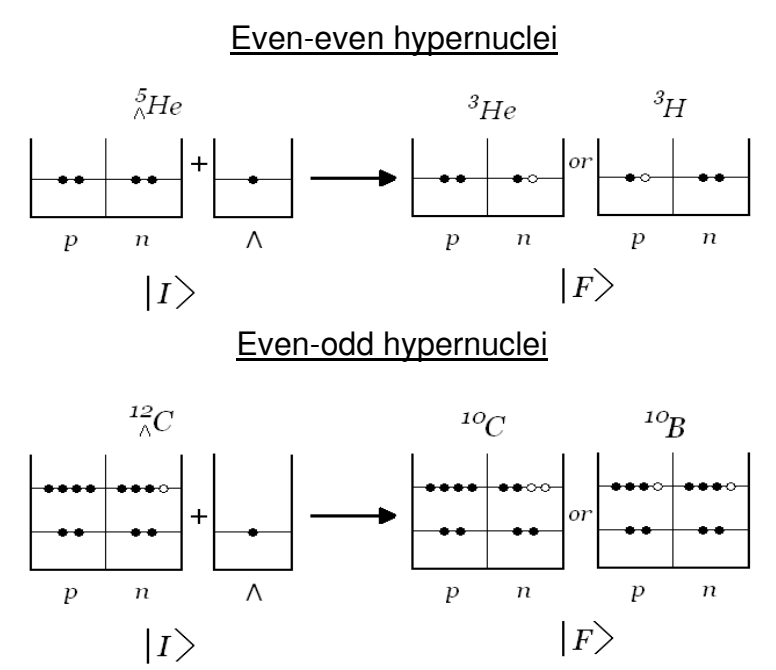

Odd-odd hypernuclei

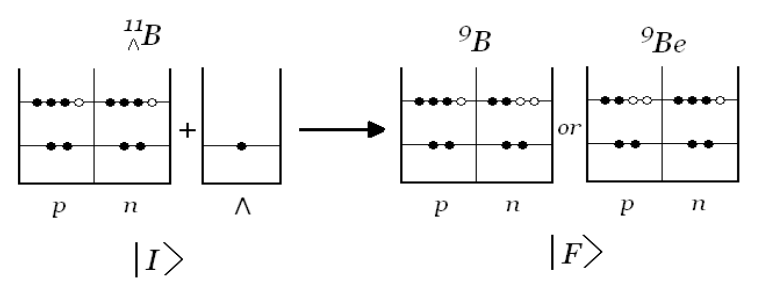

FIG. 1: Initial and final states contributing to the hypernuclear weak decay for even-even, even-odd and odd-odd core cases.

TABLE I: Numerical results for the intrinsic asymmetry parameter obtained within the full OMEM $\left(\omega_{\kappa}\right.$ is given in units of $\Gamma_{0}=2.5 \times$ $\left.10^{-6} \mathrm{eV}\right)$. See text for detailed explanation.

\begin{tabular}{c|c|c|c|c|c|c|c}
\hline \hline & ${ }_{\Lambda}^{4} \mathrm{He}$ & ${ }_{\Lambda}^{5} \mathrm{He}$ & ${ }_{\Lambda}^{11} B$ & ${ }_{\Lambda}^{12} \mathrm{C}$ & ${ }_{\Lambda}^{16} \mathrm{O}$ & ${ }_{\Lambda}^{17} \mathrm{O}$ & ${ }_{\Lambda}^{28} \mathrm{Si}$ \\
\hline$\omega_{0}\left(1 s_{1 / 2}\right)$ & 0.564 & 0.553 & 0.468 & 0.462 & 0.437 & 0.429 & 0.327 \\
$\omega_{0}\left(1 p_{3 / 2}\right)$ & - & - & 0.340 & 0.508 & 0.481 & 0.471 & 0.359 \\
$\omega_{0}\left(1 p_{1 / 2}\right)$ & - & - & - & - & 0.229 & 0.224 & 0.173 \\
$\omega_{0}\left(1 d_{5 / 2}\right)$ & - & - & - & - & - & - & 0.281 \\
\hline$\omega_{0}$ & 0.564 & 0.553 & 0.808 & 0.970 & 1.148 & 1.124 & 1.140 \\
\hline$\omega_{1}\left(1 s_{1 / 2}\right)$ & -0.298 & -0.297 & -0.258 & -0.255 & -0.242 & -0.233 & -0.178 \\
$\omega_{1}\left(1 p_{3 / 2}\right)$ & - & - & -0.169 & -0.260 & -0.248 & -0.240 & -0.186 \\
$\omega_{1}\left(1 p_{1 / 2}\right)$ & - & - & - & - & -0.119 & -0.114 & -0.090 \\
$\omega_{1}\left(1 d_{5 / 2}\right)$ & - & - & - & - & - & - & -0.139 \\
\hline$\omega_{1}$ & -0.298 & -0.297 & -0.427 & -0.515 & -0.609 & -0.587 & -0.593 \\
\hline$a_{\Lambda}$ & -0.529 & -0.538 & -0.529 & -0.530 & -0.530 & -0.522 & -0.520 \\
\hline \hline
\end{tabular}

The authors are fellows of the CONICET, CCT La Plata (Argentina) and thank the support under grant PIP 06-6159.

TABLE II: Experimental data for the NMHD asymmetry parameter.

\begin{tabular}{l|c}
\hline \hline & $a_{\Lambda}^{\exp }$ \\
\hline${ }_{\Lambda}^{4} \mathrm{He}$ & - \\
\hline${ }_{\Lambda}^{5} \mathrm{He}$ & $0.07 \pm 0.08_{-0.00}^{+0.08}[14,37]$ \\
& $0.11 \pm 0.08 \pm 0.04[15,38]$ \\
& $0.24 \pm 0.22[39]$ \\
\hline${ }_{\Lambda}^{11} B$ & $-0.16 \pm 0.28_{-0.00}^{+0.18}[37]$ \\
& $-0.20 \pm 0.26 \pm 0.04[38]$ \\
& $0.28 \pm 0.14[40]$ \\
\hline${ }_{\Lambda}^{12} \mathrm{C}$ & $-0.24 \pm 0.26_{-0.00}^{+0.08}[14]$ \\
& $-0.16 \pm 0.28_{-0.00}^{+0.18}[37]$ \\
& $-0.20 \pm 0.26 \pm 0.04[15,38]$ \\
& $0.02 \pm 0.20[40]$ \\
\hline${ }_{\Lambda}^{16} O$ & - \\
\hline${ }_{\Lambda}^{17} O$ & - \\
\hline${ }_{\Lambda}^{28} S i$ & - \\
\hline \hline
\end{tabular}

TABLE III: Numerical values of the $L=0$ coefficients contributing to $\omega_{0}$ and $\omega_{1}$ for all hypernuclei considered in the present work.

\begin{tabular}{lr|lr}
\hline \hline$\omega_{0}$ & $\omega_{1}$ & \\
\hline$c_{1}$ & 6 & $\sqrt{3} c_{1}$ & 0 \\
$c_{2}$ & 18 & $\sqrt{3} c_{2}$ & 12 \\
$c_{3}$ & 0 & $\sqrt{3} c_{3}$ & 8 \\
$c_{4}$ & 12 & $\sqrt{3} c_{4}$ & -12 \\
$c_{5}$ & 108 & $\sqrt{3} c_{5}$ & 0 \\
$c_{6}$ & -72 & $\sqrt{3} c_{6}$ & 12 \\
$c_{7}$ & 2 & $\sqrt{3} c_{7}$ & 0 \\
$c_{8}$ & 6 & $\sqrt{3} c_{8}$ & 0 \\
$c_{9}$ & 6 & $\sqrt{3} c_{9}$ & 8 \\
$c_{10}$ & -4 & $\sqrt{3} c_{10}$ & 0 \\
$c_{11}$ & 0 & $\sqrt{3} c_{11}$ & 0 \\
$c_{12}$ & 8 & $\sqrt{3} c_{12}$ & -24 \\
\hline \hline
\end{tabular}

[1] G. Garbarino, A. Parreño and A. Ramos, Phys. Rev. Lett. 91, 112501 (2003).

[2] G. Garbarino, A. Parreño and A. Ramos, Phys. Rev. C69, 054603 (2004).

[3] A. Feliciello, Nucl. Phys. A691, 170c (2001); P. Gianotti, Nucl. Phys. A691, 483c (2001).

[4] R.L. Gill, Nucl. Phys. A691, 180c (2001).
[5] S. Okada et al., Nucl. Phys. A752, 169c (2005).

[6] B.H. Kang et al., Phys. Rev. Lett. 96, 062301 (2006).

[7] M.J. Kim et al., Phys. Lett. B641, 28 (2006).

[8] J.D. Parker et al., Phys. Rev. C76 035501 (2007).

[9] H. Bhang et al., Eur. Phys. J. A33, 259 (2007).

[10] M. Agnello et al., Nucl. Phys. A804, 151 (2008).

[11] S. Ajimura et al., Phys. Lett. B282, 293 (1992). 
TABLE IV: Numerical values of the $L=1$ coefficients contributing to $\omega_{0}$ and $\omega_{1}$ for ${ }_{\Lambda}^{11} \mathrm{~B}$ hypernucleus.

\begin{tabular}{c|r|r}
\hline \hline $\mathrm{i}$ & $r_{i}$ & $\sqrt{3} \mathrm{r}_{i}$ \\
\hline 1 & $\frac{35}{6}$ & $-\frac{4}{3}$ \\
2 & $\frac{27}{2}$ & $\frac{35}{3}$ \\
3 & 4 & 10 \\
4 & $\frac{23}{3}$ & -9 \\
5 & 69 & 4 \\
6 & -46 & 9 \\
7 & $\frac{3}{2}$ & $\frac{8}{3}$ \\
8 & $\frac{73}{18}$ & $\frac{8}{3}$ \\
9 & $\frac{73}{18}$ & 6 \\
10 & $-\frac{19}{9}$ & -8 \\
11 & $\frac{4}{9}$ & -8 \\
12 & $\frac{50}{9}$ & -18 \\
\hline
\end{tabular}

[12] S. Ajimura et al., Phys. Rev. Lett. 84, 4052 (2000).

[13] T. Maruta et al., Nucl. Phys. A754, 168c (2005). See also nuclex/0402017.

[14] H. Bhang et al., Nucl. Phys. A754, 144c (2005).

[15] H. Outa et al., Nucl. Phys. A754, 157c (2005).

[16] K. Sasaki, T. Inoue and M. Oka, Nucl. Phys. A669, 331 (2000), Erratum-ibid A678, 455 (2000).

[17] J. F. Dubach, G. B. Feldman, B. R. Holstein and L. de la Torre, Ann. Phys. (N.Y.) 249, 146 (1996).

[18] A. Parreño, A. Ramos and C. Bennhold, Phys. Rev. C56, 339 (1997) and references therein.

[19] A. Parreño and A. Ramos, Phys. Rev. C65, 015204 (2001).

[20] C. Barbero, C. de Conti, A.P. Galeao and F. Krmpotić, Nucl. Phys. A726, 267 (2003).

[21] K. Itonaga, T. Ueda and T. Motoba, Phys. Rev. C 65, 034617 (2002); K. Itonaga, T. Motoba and T. Ueda, Electrophoto Production of Strangeness on Nuand Nuclei (Sendai03), K. Maeda,
H. Tamura, S.N. nakamura and O. Hashimoto eds., World Scientific (2004) pp. 397-402.

[22] K. Sasaki, T. Inoue and M. Oka, Nucl. Phys. A702, 477 (2002).

[23] C. Barbero, A.P. Galeao and F. Krmpotić, Phys. Rev. C72, $035210(2005)$

[24] C. Barbero and A. Mariano, Phys. Rev. C73, 024309 (2006).

[25] C. Barbero, A.P. Galeao and F. Krmpotić, Braz. J. Phys. 34, 822 (2004).

[26] C. Barbero, A.P. Galeao and F. Krmpotić, Braz. J. Phys. 36, 1357 (2006).

[27] W. M. Alberico and G. Garbarino, Phys. Rep. 369, 1 (2002).

[28] W. M. Alberico, G. Garbarino, A. Parreño and A. Ramos, Phys. Rev. Lett. 94, 082501 (2005).

[29] A. Parreño, C. Bennhold and B.R. Holstein, Phys. Rev. C70, 051601 (2004).

[30] K. Sasaki, M. Izaki and M. Oka, Phys. Rev. C71, 035502 (2005).

[31] C. Chumillas, G. Garbarino, A. Parrenõ and A. Ramos, Phys. Lett. B657, 180 (2007); C. Chumillas, G. Garbarino, A. Parrenõ and A. Ramos, Nucl. Phys. A804, 162 (2008).

[32] K. Itonaga, T. Motoba and T. Ueda, Phys. Rev. C 77, 044605 (2008).

[33] C. Barbero, A.P. Galeao and F. Krmpotić Phys. Rev. C76 054321 (2007).

[34] H. Nabetani, T. Ogaito, T. Sato and T. Kishimoto, Phys. Rev. C60, 017001 (1999).

[35] A. de Shalit and I. Talmi, Nuclear Shell Theory, Academic Press New York and London, p. 189 (1963).

[36] C. Barbero, D. Horvat, F. Krmpotić, T.T.S. Kuo , Z. Narančić and D. Tadić Phys. Rev. C66 055209 (2002).

[37] T. Maruta, Ph. D. thesis, KEK Report 2006-1, 2006.

[38] T. Maruta et al., nucl-ex/0402017; ibid, Nucl. Phys. A754, 168c (2005).

[39] S. Ajimura et al., Phys. Rev. Lett. 84, 4052 (2000).

[40] S. Ajimura et al., Phys. Lett. B282, 293 (1992).

[41] M. M. Block and H. H. Dalitz, Phys. Rev. Lett. 11, 96 (1963).

[42] J. B. Adams, Phys. Rev. 156, 832 (1967). (Cf. correction pointed out in Ref. [43].)

[43] B.H.J. McKellar and B.F. Gibson, Phys. Rev. C30, 322 (1984). 